\title{
The Correlation between Plasma Concentration of Aripiprazole and Clinical Efficacy in the Treatment of Children and Adolescent Patients with Schizophrenia
}

\author{
Shuzhen Jiang ${ }^{1}$, Kun $\mathrm{Yan}^{2}$, Yonghui $\mathrm{Fu}^{3}$, Bin Yu ${ }^{2}$, Yuanjian Yang ${ }^{2^{*}}$ \\ ${ }^{1}$ Department of Pharmacy, Jiangxi Mental Hospital, Nanchang 330029, China; \\ ${ }^{2}$ Medical Experimental Center, Jiangxi Mental Hospital, China; \\ ${ }^{3}$ Department of Pediatric Psychiatry, Jiangxi Mental Hospital, China.
}

*Correspondence: Yuanjian Yang, Email: yuanjimyang@yeah.net .

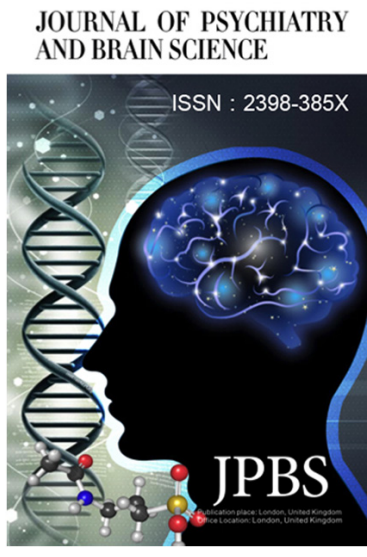

http://jpbs.qingres.com

GOPEN ACCESS

DOI: 10.20900/jpbs.20170020

Received: November 9, 2017

Accepted: November 9, 2017

Published: December 25, 2017

Copyright: ๑2017 Cain et al. This is an open access article distributed under the terms of the Creative Commons Attribution License, which permits unrestricted use, distribution, and reproduction in any medium, provided the original author and source are credited.

\begin{abstract}
Objective: To investigate the correlation between plasma drug concentration of aripiprazole and its clinical efficacy in the treatment of children and adolescent patients with schizophrenia.

Methods: 27 cases of children and adolescent patients with schizophrenia received oral non-fixed dosage of aripiprazole for 8 weeks. The plasma concentration of aripiprazole was determined with High Performance Liquid Chromatography (HPLC). The psychotic symptoms of patients were evaluated with Positive and Negative Syndrome Scale (PANSS) and the adverse reactions were assessed with Treatment Emergent Symptom Scale (TESS).
\end{abstract}

Results: There was no correlation between the plasma concentration of aripiprazole and the reduction rate of PANSS scores at the end of first week $(r=0.201, p>0.05)$. A positive correlation between the plasma concentration of aripiprazole and the reduction rate of PANSS scores was found at the end of second week $(r=0.352, p=0.055)$, and significant correlations were observed at the end of both fourth and eighth weeks $(r>0.503, p<0.05)$. No relationship was found between the oral dosage of aripiprazole and the clinical efficacy. The mean concentration of aripiprazole in effective group was higher than that of ineffective group. The lowest effective concentration of aripiprazole was $285.2 \mathrm{ng} / \mathrm{ml}$ and the concentration threshold occurring side effects was $440 \mathrm{ng} / \mathrm{ml}$. 
Conclusion: The plasma concentration of aripiprazole is correlated with its clinical efficacy in the treatment of children and adolescent patients with schizophrenia symptoms, and the suitable concentration range is $285.2 \sim 440 \mathrm{ng} / \mathrm{ml}$.

Key Words: Schizophrenia; Children and adolescent patients; Aripiprazole; plasma concentration; Clinical efficacy

\section{INTRODUCTION}

Although childhood-onset schizophrenia is rare, it is relatively common for adolescent-onset schizophrenia ${ }^{[1-2]}$. Safe and effective treatments are the key for the youths. Aripiprazole is a secondgeneration antipsychotic drug that is differentiated from traditional antipsychotics by its low levels of unwanted side effects, and in general increased safety $^{[3-4]}$. In particular, aripiprazole is a dopamine partial agonist that has been shown to be efficacious in the treatment of positive and negative symptoms in acute and long-term treatment of schizophrenia in adults at doses of 10 to $30 \mathrm{mg} /$ day $^{[5-6]}$. In 2007, the FDA officially approved aripiprazole as a drug for the treatment of schizophrenia in children and adolescents aged 13 to 17 years. Studies have shown that aripiprazole treatment of schizophrenia in children and adolescents is relative effective and safe ${ }^{[7-8]}$. It has been extensively investigated the correlation between steady-state plasma concentration of aripiprazole and clinical outcomes in the treatment of adult schizophrenia (referred to as plasma concentration) ${ }^{[9-10]}$. However, the correlation between plasma concentration of aripiprazole and clinical outcomes when used on children and adolescents remains uncertain. In this study, we tested the plasma concentration of aripiprazole in children and adolescents patients and found a close correlation between the clinical outcomes and the plasma concentration. Our findings shed lights on the safe and effective treatment with aripiprazole in children and adolescents with schizophrenia.

\section{MATERIAL AND METHODS}

\subsection{Participants}

Participants comprised 27 patients diagnosed with schizophrenia present in the Jiangxi Mental Hospital between July 2015 and June 2016. Of the 27 patients, 14 patients were male and 13 were female (Avg. age $15 \pm 2$ ). The duration of illness ranged from 1 to 42 months with a median of 5 months.

Eligible subjects met the following inclusion criteria: 1) primary diagnosis of schizophrenia and confirmation of the schizophrenia diagnosis by an adequately trained clinician (e.g., child psychiatrist) at the time of screening, by means of the International Statistical Classification of diseases and related health problems 10th revision (ICD-10); 2) age 12 to 17 years inclusive; 3) Han nationality; 4) a baseline a Positive and Negative Syndrome Scale (PANSS) score of 60 or higher; 5) written, informed consent was included.

Exclusion criteria: 1) other types of psychiatric disorders, alcohol or drug abuse; 2) diagnosed central nervous system diseases such as cancer, epilepsy, traumatic brain injury history; 3) a variety of somatic diseases such as infection, diabetes, heart disease; 4) history of serious allergic reaction. This study was pre-approved by the Ethics Committee of Jiangxi Mental Hospital.

\subsection{Medication}

After 3 to 5 days of drug washout period, the patients were started to receive oral aripiprazole at an initial dose of $5 \mathrm{mg} /$ day (split into twice a day). Based on the individual tolerance the dose of the drug was gradually increased every 3-5 days by an increment of $5 \mathrm{mg}$ with a maximal daily dose of no more than $30 \mathrm{mg}$. The course of treatment lasted for 8 weeks. During the study, patients were treated with benzodiazepine for insomnia; metoprolol for tachycardia, and Trihexyphenidyl for the emergence of extrapyramidal reactions.

\subsection{Clinical assessment}

1) Efficiency assessment: Assessments were performed at baseline and at the end of 1 week, 2 weeks, 4 weeks and 8 weeks respectively. Decrease at least 50 \% in Positive and Negative Syndrome Scale (PANSS) total score is deemed as positive response to treatment, while less than $50 \%$ is deemed as negative response to treatment.

2) Safety and Tolerability Assessments: The measurement of side effects was based on the score of the Treatment Emergent Symptom Scale (TESS). The Kappa value is greater than 0.8 . 


\subsection{Aripiprazole plasma drug concentration Assay ${ }^{[11]}$}

Blood samples were collected at 6:00 am on the morning of the 1st, 2nd, 4th and 8th weekend after taking the drug. After centrifugation, the supernatant serum was collected and stored in a Freezer set at $-80 \quad{ }^{\circ} \mathrm{C}$ for storage. Aripiprazole plasma drug concentrations were determined using high performance liquid chromatography (HPLC). Aripiprazole used for treatment was purchased from the China Pharmaceutical and Biological Products (purity greater than $99.8 \%$ ). The minimal plasma Aripiprazole concentration that can be detected was $5 \mathrm{ng} / \mathrm{ml}$, and the minimum dose was $2.5 \mathrm{ng}$. The average extraction recovery was $81 \%$ and the recovery rate was $96 \sim 99 \%$.

\subsection{Statistical Analyses}

Statistical analysis was performed using SPSS 18.0 statistical software. Data shown as mean \pm standard deviation $( \pm s)$. Chi-square test or paired t-test was used to for data comparison between groups. Person correlation analysis was used to test the correlation of measurement data. A nominal overall significance level of 0.05 (two-tailed) was used in testing the statistical significance of these comparisons.

\section{RESULTS}

\subsection{Comparison of PANSS scores before and after aripiprazole treatment}

Table 1. shows the scores of PANSS in children and adolescents with schizophrenia before and after aripiprazole treatment. The PANSS total score, positive symptom score, and general psychopathology score showed noticeable decrease starting from the second weekend $(p<0.05)$. Compared with the baseline, PANSS total score and each factor score showed a significant reduction from week $4(p<0.05$ or 0.01$)$.

Table 1. PANSS total scores and each factor score (Mean scores $\pm \mathbf{s d}$ ) comparisons before and after treatment.

\begin{tabular}{lcccc}
\hline Duration (weeks) & Total score & Positive score & Negative score & $\begin{array}{l}\text { General pathological } \\
\text { symptoms score }\end{array}$ \\
\hline 0 (Baseline) & $83.5 \pm 9.2$ & $23.5 \pm 4.2$ & $20.2 \pm 5.4$ & $39.8 \pm 6.6$ \\
1 & $79.4 \pm 8.9$ & $21.3 \pm 3.8$ & $19.1 \pm 6.5$ & $38.9 \pm 7.1$ \\
2 & $70.2 \pm 5.5^{1)}$ & $17.8 \pm 5.2^{1)}$ & $18.0 \pm 3.9$ & $34.4 \pm 6.3^{1)}$ \\
4 & $61.3 \pm 7.6^{1)}$ & $15.9 \pm 5.2^{2)}$ & $16.6 \pm 4.3^{1)}$ & $28.8 \pm 5.7^{2)}$ \\
8 & $51.1 \pm 4.9^{2)}$ & $12.6 \pm 5.2^{2)}$ & $13.8 \pm 5.5^{2)}$ & $24.6 \pm 4.2^{2)}$ \\
\hline
\end{tabular}

Note: 1) $p<0.05 ;$ 2) $p<0.01$ compared with the baseline score.

\subsection{Correlation Analysis of aripiprazole plasma drug concentrations, drug doses and dose responses}

Table 2. shows the plasma drug concentration, drug dose, PANSS total score reduction rate from the patients treated with aripiprazole for 1 , 2, 4 or 8 weeks. There was a strong correlation between aripiprazole plasma concentration and medication dose $(p<0.05)$. Interestingly, plasma concentration of aripiprazole at the end of the first week of treatment had no correlation with PANSS reduction rate $(r=0.201, p>0.05)$, whereas, after two weeks of treatment a decrease of PANSS was observed ( $r=0.352, p=0.055)$. Moreover, there was a significant positive correlation between the 
plasma concentration of aripiprazole and the PANSS reduction rate at the 4 th and 8 th week $(r>0.503, p<$ $0.05)$. Furthermore, correlation analysis of the dose of aripiprazole with the PANSS cutoff rate suggested that there was no significant correlation between the dose of aripiprazole and PANSS $(p>0.05)$, indicating that increasing the dose of the drug may not necessarily improve the clinical curative effect.

Table 2. Correlation between aripiprazole plasma drug concentration and drug dose and the PANSS reduction rate.

\begin{tabular}{|c|c|c|c|c|c|c|c|}
\hline $\begin{array}{l}\text { Duration } \\
\text { (week) }\end{array}$ & $\begin{array}{l}\text { Plasma } \\
\text { concentration } \\
(\mathrm{ng} / \mathrm{ml})\end{array}$ & $\begin{array}{l}\text { Drug dose } \\
\text { (mg) }\end{array}$ & $\begin{array}{l}\text { PANSS } \\
\text { reduction(\%) }\end{array}$ & r1 & P1 & r2 & P2 \\
\hline 1 & $156.5 \pm 46.5$ & $7.7 \pm 2.6$ & $7.4 \pm 3.3$ & 0.157 & 0.049 & 0.201 & 0.092 \\
\hline 2 & $246.7 \pm 83.5$ & $14.1 \pm 3.8$ & $24.0 \pm 7.6$ & 0.261 & 0.012 & 0.352 & 0.055 \\
\hline 4 & $311.4 \pm 108.2$ & $17.3 \pm 2.6$ & $40.3 \pm 15.1$ & 0.325 & 0.008 & 0.503 & 0.022 \\
\hline 8 & $341.2 \pm 127.7$ & $20.5 \pm 3.5$ & $58.4 \pm 17.2$ & 0.332 & 0.006 & 0.608 & 0.003 \\
\hline
\end{tabular}

$\mathrm{r} 1$ indicates the correlation coefficient between plasma concentration and drug dose; $r 2$ indicates the correlation coefficient between plasma concentration and PANSS reduction.

\subsection{Analysis of the minimum effective plasma drug concentration}

Among 27 patients treated with aripiprazole for 8 weeks, 19 patients showed positive responses (PANSS reduction $\geq 50 \%$ ) and 8 patients showed less responsive, which leads to a response rate of $70.4 \%$ (19/27). Plasma drug concentration comparison suggested that the concentration of the effective group in the $2 \mathrm{nd}$, 4th and 8 th week were significantly higher than that of the ineffective group $(p<0.05)$ (Table 3$)$. Based on the correlation between drug concentration and clinical outcomes, the optimal cutoff value of blood concentration at the end of the 8th week was analyzed by ROC curve. The results showed that the best cutoff value of $285.2 \mathrm{ng} / \mathrm{ml}$ plasma concentration, that is, the minimum effective plasma concentration.

Table 3. The plasma drug concentrations of effective and non-effective groups at different time points (Mean scores $\pm \mathrm{sd}$; $\mathrm{ng} / \mathrm{ml}$ ).

\begin{tabular}{lllll}
\hline Group & 1st week & 2nd week & 4th week & 8th week \\
\hline Effective group & $169.3 \pm 52.9$ & $278.6 \pm 71.5$ & $347.1 \pm 64.6$ & $378.3 \pm 120.1$ \\
Non-effective group & $126.2 \pm 60.1$ & $181.1 \pm 82.3$ & $225.6 \pm 70.7$ & $253.2 \pm 104.6$ \\
$\mathrm{t}$ & 1.357 & 2.018 & 2.148 & 2.560 \\
$\mathrm{P}$ & 0.189 & 0.054 & 0.042 & 0.017 \\
\hline
\end{tabular}




\subsection{Analysis of adverse drug reactions}

The incidence of adverse reactions was $33.3 \%$ $(9 / 27)$ in 27 patients treated with aripiprazole, including extrapyramidal reactions (4), restlessness (1), nausea (1), Lethargy (1), insomnia (1), abnormal liver function (1). Adverse drug reactions generally appeared after 2 to 3 weeks' medication or increasing the drug doses. ROC curve was used to find out the threshold between aripiprazole plasma drug concentration and adverse drug reactions. Results showed that the optimal plasma concentration cutoff value was $440 \mathrm{ng} / \mathrm{ml}$, that is, plasma concentration greater than this value is more likely to increase the adverse drug reactions.

\section{DISCUSSION}

Previously, aripiprazole has been shown to be a partial agonist at the D2 dopamine receptor ${ }^{[4]}$.The stabilizing effect of aripiprazole on the dopamine system was attributed to its targeting of presynaptic (auto-receptors) and post-synaptic D2 receptors [3]. Therefore, it is effective to positive symptoms, negative symptoms as well as symptoms of cognitive impairments. In addition, aripiprazole is classified as a medication with significant $5 \mathrm{HT} 2 \mathrm{~A}-$ antagonism, and with still other additional secondary binding characteristics such as 5-HT2C, 5-HT7, epinephrine $\alpha 1$ and histamine $\mathrm{H} 1$ receptors. Its unique mechanism of action makes aripiprazole as a favorable clinical treatment choice over others, i.e. high efficacy, fewer extrapyramidal reactions (EPS), does not cause weight gain, does not cause the incensement of prolactin (PRL) ${ }^{[3,12-13]}$. Consistently, our results showed that the overall response rate of aripiprazole treatment was 70.4 $\%$, which was similar to that reported by Beijing Anding Hospital ${ }^{[7]}$. The PANSS total score, positive symptom score and general psychopathology score decreased significantly from baseline level starting from the second week of treatment, and the negative symptom score began to decrease significantly from the end of the fourth week of treatment until the end of the study.

Current studies on aripiprazole treatment of adult schizophrenia have found a strong correlation between plasma concentration and clinical efficacy ${ }^{[9-11]}$. Our study focused on the aripiprazole treatment to schizophrenia in children and adolescents and found a similar positive correlation with the PANSS reduction rate at the end of weeks 4 and 8 . Comparison of the plasma concentration of aripiprazole between the effective group and the ineffective group also found that the plasma concentration of the effective group was significantly higher than that of the ineffective group at the 4th and 8th week. Together, these results suggested that there is strong correlation between plasma concentration and clinical efficacy in children and adolescents with schizophrenia with aripiprazole treatment. Because of the individual differences due to heredity and environment, taking the same dose of drug does not necessarily result in the same level of plasma concentrations. Indeed, our study found no significant correlation between the patient's medication dose and the PANSS scale reduction rate. Overall, our findings suggested that a closer monitor of plasma drug concentration will help better guide the dose of the drug to reduce the blindness of drug adjustment, and in turn patients would receive the best outcomes.

Studies have shown that the optimal plasma concentration of aripiprazole treatment to adult schizophrenia is in the range of $363-550 \mathrm{ng} / \mathrm{ml}$ for a better efficiency ${ }^{9]}$. In this study, we found that the minimum effective plasma drug concentration of aripiprazole for the treatment of schizophrenia in children and adolescents is $285.2 \mathrm{ng} / \mathrm{ml}$, while the optimal plasma concentration cutoff from adverse drug reactions is $440 \mathrm{ng} / \mathrm{ml}$, suggesting that the optimal aripiprazole treatment of schizophrenia in children and adolescents is in the range of 285.2$440 \mathrm{ng} / \mathrm{ml}$. Therefore, the suggested plasma drug concentrations dosage for schizophrenia in children and adolescents is significantly lower than that of adult patients, and we speculate that it is likely due to the relatively larger liver and higher metabolism rate of children and adolescents as compared to the adults. In addition, this observation indicates that plasma drug concentration can be a useful guideline for the adjustment of the drug treatment dose.

Out study found that aripiprazole treatment of schizophrenia in children and adolescents has an overall efficiency rate of $70.4 \%$, with an incidence rate of $33.3 \%$ and similar types of adverse drug reactions as adult patients, suggesting the a favorable safety and efficacy profile of the aripiprazole as a treatment to children and adolescents. Future study could recruit a larger sample size for a more comprehensive examination of the drug efficacy. Also, a better understanding of the drug to the treatment of children and adolescents can be achieved by measuring the concentration of aripiprazole and its downstream active metabolites with the body mass index taking into account. 


\section{ACKNOWLEDGMENTS}

This work was funded by grants from the Project of Nanchang Science and Technology Bureau of China (2014SFZC017) and the Natural Science Foundation of Jiangxi Province of China (20171BAB205020).

\section{REFERENCES}

1. Burd L, Kerbeshian J. A North Dakota prevalence study of schizophrenia presenting in childhood. J Am Acad Child Adolesc Psychiatry. 1987; 26:347-350.

2. Thomsen PH. Schizophrenia with childhood and adolescent onset-a nationwide register-based study. Acta Psychiatr Scand. 1996; 94:187-193.

3. Marder SR, McQuade RD, Stock E, Kaplita S, Marcus R, Safferman AZ, Saha A, Ali M, Iwamoto T. Aripiprazole in the treatment of schizophrenia: safety and tolerability in shortterm, placebo-controlled trials. Schizophr Res. 2003 Jun 1; 61(2-3):123-136.

4. Stip E, Tourjman V. Aripiprazole in schizophrenia and schizoaffective disorder: A review. Clin Ther. 2010; 32 Suppl 1:S3-20.

5. Cutler AJ, Marcus RN, Hardy SA, O'Donnell A, Carson WH, McQuade RD. The efficacy and safety of lower doses of aripiprazole for the treatment of patients with acute exacerbation of schizophrenia. CNS Spectr. 2006; 11:691-702.

6. Potkin SG, Saha AR, Kujawa MJ, Carson WH, Ali M, Stock E, Stringfellow J, Ingenito G, Marder SR. Aripiprazole, an antipsychotic with a novel mechanism of action, and risperidone vs placebo in patients with schizophrenia and schizoaffective disorder. Arch Gen Psychiatry. 2003; 60:681-690.

7. Chen $X$, Zheng Y.Efficacy and tolerability of aripiprazole on childhood-onset schizophrenia. Journal of Neuroscience and Mental Health. 2010; 10(1):66-68.

8. Findling RL, Robb A, Nyilas M, Forbes RA, Jin $\mathrm{N}$, Ivanova S, Marcus R, McQuade RD, Iwamoto $\mathrm{T}$, Carson WH. A multiple-center, randomized, double-blind, placebo-controlled study of oral aripiprazole for treatment of adolescents with schizophrenia. Am J Psychiatry. 2008; 165(11):1432-1441.

9. Li JH, Zhong $\mathrm{H}$, Shen WM, et al. Study on the relationship between the concentration of aripiprazole in the treatment of schizophrenia and the clinical effect. Chin J Clin Pharm Therap. 2011; (01):76-81.

10. Lin SK, Chen CK, Liu YL. Aripiprazole and dehydroaripiprazole plasma concentrations and clinical responses in patients with schizophrenia. J Clin Psychopharmacol. 2011; 31(6):758-762.

11. Zhan HY, Hu MR, Yu B, et al. Relationship between serum concentration and clinical efficacy of aripiprazole in the treatment of patients with first episode schizophrenia. Jiangxi Medical Journal. 2013; (11):958-960.

12. Davies MA, Sheffler DJ, Roth BL. Aripiprazole: a novel atypical antipsychotic drug with a uniquely robust pharmacology. CNS Drug Rev. 2004; 10(4):317-336.

13. Zhou P, Liu LQ, Hao JF, et al. Study of aripiprazole in preventing hyperprolactinemia induced by antipsychotics in patients with schizophrenia. J Int Psychl. 2014; (02):68-72. 